\title{
La Communication Numérique Sur La RSO Dans Le Milieu Hospitalier
}

\author{
Jebari Bouchra (Doctorante) \\ Faculté des Sciences de l'Education/ \\ Université Mohammed V de Rabat, Maroc
}

doi: 10.19044/esj.2017.v13n8p134 URL:http://dx.doi.org/10.19044/esj.2017.v13n8p134

\begin{abstract}
Mainly associated with the private sector, Corporate Social Responsibility -CSR- can also play a big and important role for public organizations; regardless of their size, type, orientation(s) or mission(s), these organizations tend to pursue more and more effective initiatives in order to be socially responsible. In health sector, hospital centers are organizations where "social" is part of their basic missions, two more aspects "environmental" and "economic" can be added in order to establish CSR basis, combining these aspects through an effective communication should be at the heart of any CSR strategy. In order to study CSR content communicated online through official moroccan hospital centers' websites and social media, we conducted this study by using different indicators from various analysis grids related to the key areas of our research (CSR, health and communication). Opting for digital communication via websites and social media can be a useful and practical way to help hospital centers be socially responsible.
\end{abstract}

Keywords: CSR, CSR communication, hospital center

\section{Résumé}

A l'instar des entreprises reconnues pour leur engagement dans des démarches de responsabilité sociétale (RSE), les organismes publics ont entamé eux aussi au cours des dernières années des pas-grands ou modestes, en fonction de la taille de l'organisation - dans le domaine de la responsabilité sociétale. Non loin de l'aspect social dont jouit la réputation des organismes de santé, deux autres grands aspects, peuvent s'y ajouter, il s'agit des aspects: environnemental et économique, afin de constituer le socle d'une responsabilité sociétale auprès d'une organisation de grande taille: le centre hospitalier. L'intégration de ces différentes pratiques nécessite le développement de stratégies de qualité notamment en terme de 
communication. Ceci dit, et afin de mieux connaitre l'approche communication RSO utilisée par chaque centre hospitalier marocain et plus particulièrement sa communication numérique via le site web, nous avons réalisé la présente étude à l'aide d’indicateurs issus de diverses grilles d'analyse et relatifs aux domaines clés de notre recherche (RSO, santé, et communication). Cet article analyse également la présence de ces centres hospitaliers sur les réseaux sociaux en tenant compte des informations portant sur leur présentation institutionnelle et les facteurs liés à la RSO. Plaider continuellement, via le digital, pour la cause de la responsabilité sociétale, permettra aux centres hospitaliers marocains de se doter de l’image d'un centre hospitalier communiquant et responsable.

Mots clés: RSO, communication RSO, centre hospitalier

\section{Introduction}

La notion de Responsabilité Sociétale est souvent associée de manière étroite aux Entreprises (RSE), et de manière plus générale aux Organisations (RSO), divers secteurs pouvant être des terrains de mise en pratique de ce concept.

La santé est l'un des secteurs reconnus comme stratégiques par nature, dont la responsabilité sociétale occupe une place peu visible, malgré le fait qu'elle soit étroitement liée à ce secteur. En effet, le concept de RSO recouvre les valeurs défendues par les établissements publics de santé et médicosociaux que sont l'humanisme, l'accessibilité, la solidarité, l'universalité et l'égalité (Martel, 2014).

Selon l'Organisation Mondiale de la Santé (OMS) « la responsabilité sociale en faveur de la santé trouve son expression dans les mesures que les décideurs des secteurs public et privé prennent pour mettre en œuvre des politiques et des pratiques qui assurent la promotion et la protection de la santé »; cette promotion se résume en un besoin d'amélioration de la santé sans nuire à la santé des autres, la protection de l'environnement du citoyen et le développement des politiques sous un principe d'équité. (Déclaration de Jakarta sur la promotion de la santé au XXIe siècle,1997).

Afin de hisser la visibilité de la responsabilité sociétale dans le secteur de la santé, demeurée jusqu'à ce jour modeste et peu communiquée, nous allons approcher la notion de communication RSO à travers une étude sur la communication numérique via les sites web et les réseaux sociaux officiels des centres hospitaliers marocains.

Il s'agit de l'un des types de communication les plus répandus, par lequel la plupart des organismes ont développé leur présence, d'autant plus qu'il permet d'atteindre une population plus large,certains responsables et managers proactifs de la RSO utilisent de plus en plus les sites web à des fins 
de communication, que ça soit pour des raisons commerciales ou altruistes (Parker et al. 2010).

Le milieu de la santé et des services sociaux n’échappe pas à cette tendance, comme tout autre domaine d'ailleurs, les changements technologiques sont de plus en plus rapides et l'utilisation des ressources du réseau internet s’accentue progressivement (Equipe régionale en documentation-régie régionale de la santé et des services sociaux montréalcentre, 1997).

Au Maroc, le nombre d'internautes ne cesse d'augmenter; en 2014, le royaume a enregistré un million de nouveaux internautes portant leurs nombre à 17,3 millions, soit l'équivalent de 56,8\% de la population (Agence nationale de réglementation des télécommunications, 2014).

Il est à noter que chaque organisation créée devra se doter de son propre site web qui représente sa vitrine dans le monde du web, et naturellement, cette vitrine doit refléter le plus objectivement possible ses missions et ses activités.

Cet espace économique qui a empiété sur les autres médias traditionnels, demeure l'un des modes de communication institutionnelle privilégié des organisations qui souhaiteraient communiquer des renseignements envers leurs publics. Les réseaux sociaux sont sollicités également dans un cadre institutionnel mais avec un taux beaucoup plus faible.

Les cinq centres hospitaliers du Maroc ont chacun un site web officiel, et sont également présents sur les réseaux sociaux.

Un centre hospitalier est un établissement public doté de la personnalité morale et de l'autonomie financière, et est soumis à la tutelle de l'état (Dahir n 1.82.5, 1983) .

Il constitue l'un des établissements d'un centre hospitalier et universitaire (CHU) au côté des facultés de médecine et de pharmacie, des facultés de médecine dentaire, et des formations hospitalières militaires en plus des établissements de soins créés par des organisations à but non lucratif, après avis d'une commission. (par exemple le Centre Hospitalier et Universitaire Ibn-Sina à Rabat comprend les établissements suivants: (B.O $\left.\mathrm{N}^{\circ} 5388,2006\right)$

$\checkmark$ le centre hospitalier Ibn Sina à Rabat;

$\checkmark$ l'hôpital militaire d'instruction Mohammed V à Rabat;

$\checkmark$ la faculté de médecine et de pharmacie à Rabat;

$\checkmark$ la faculté de médecine dentaire à Rabat; ;

$\checkmark$ l'hôpital Cheikh Zaid Ibn Sultan à Rabat.

Les établissements des CHU organisent, conjointement, leurs services en centres de soins, d'enseignement et de recherche, où sont 
dispensés les enseignements médicaux, pharmaceutiques et odontologiques, théoriques et pratiques (Décret n²-04-776,2005) .

Chaque centre hospitalier a pour missions (Dahir $\left.n^{\circ} 1.82 .5,1983\right)$ :

$\checkmark$ de dispenser les soins médicaux ;

$\checkmark$ d'effectuer des travaux de recherche médicale, dans le strict respect de l'intégrité physique et morale et de la dignité des malades ;

$\checkmark$ de participer à l'enseignement clinique universitaire et postuniversitaire médical et pharmaceutique et à la formation du personnel para-médical ;

$\checkmark$ de concourir à la réalisation des objectifs fixés en matière de santé publique par l'état.

Chacun des centres hospitaliers marocains offre plusieurs formations hospitalières concourant à la réalisation des missions citées auparavant :

\begin{tabular}{|c|c|c|c|}
\hline $\begin{array}{c}\text { Centres } \\
\text { Hospitaliers } \\
(\mathrm{CH})\end{array}$ & Siège & $\begin{array}{c}\text { Nombre de } \\
\text { formations } \\
\text { hospitalières }\end{array}$ & Formations Hospitalières \\
\hline CH Ibn-Sina & Rabat & 10 & $\begin{array}{c}\text {-Hôpital Ibn Sina ; } \\
\text {-Hôpital des Spécialités Rabat ; } \\
\text {-Hôpital d’Enfants Rabat ; } \\
\text {-Institut National d’Oncologie ; } \\
\text {-Maternité Souissi ; } \\
\text {-Centre National de Santé Reproductrice ; } \\
\text {-Hôpital Moulay Youssef ; } \\
\text {-Centre de Consultations et de Traitements } \\
\text { Dentaires ; } \\
\text {-Hôpital El Ayachi ; } \\
\text {-Hôpital Ar-Razi Salé . }\end{array}$ \\
\hline CH Ibn-Rochd & Casablanca & 4 & $\begin{array}{c}\text {-Hôpital Ibn Rochd ; } \\
\text {-Hôpital d’Enfants ; } \\
\text {-Hôpital du } 20 \text { Août ; } \\
\text {-Centre de Consultations et de Traitements } \\
\text { Dentaires. }\end{array}$ \\
\hline $\begin{array}{c}\text { CH Mohammed } \\
\text { VI }\end{array}$ & Marrakech & 5 & $\begin{array}{l}\text {-Hôpital Ibn Tofail ; } \\
\text {-Hôpital Mère et Enfant ; } \\
\text {-Centre d’Oncologie Hématologie ; } \\
\text {-Hôpital Ibn Nafis ; } \\
\text {-Hôpital Ar-Razzi . }\end{array}$ \\
\hline CH Hassan II & Fès & 5 & $\begin{array}{c}\text {-Hôpital des Spécialités ; } \\
\text {-Hôpital Mère et Enfant ; } \\
\text {-Hôpital d’Oncologie et de Médecine Nucléaire ; } \\
\text {-Hôpital Omar Drissi ; } \\
\text {-Hôpital Ibn Al Hassan. }\end{array}$ \\
\hline $\begin{array}{c}\text { CH Mohammed } \\
\text { VI }\end{array}$ & Oujda & 5 & $\begin{array}{c}\text {-Hôpital des Spécialités ; } \\
\text {-Hôpital Mère-Enfant ; } \\
\text {-Centre des Brulés ; } \\
\text {-Centre d’Oncologie Hassan II ; } \\
\text {-Hôpital de la Santé Mentale et des Maladies } \\
\text { Psychiatriques. }\end{array}$ \\
\hline
\end{tabular}

Tableau 1. Liste des formations hospitalières composant chaque centre hospitalier au Maroc 
Au total, le Maroc compte jusqu'à date -l'année 2017- cinq centres hospitaliers opérationnels, dont le nombre de formations hospitalières diffère-voir le tableau ci-dessus-, trois nouveaux centres hospitaliers sont en cours de réalisation dans trois autres villes marocaines: Tanger, Agadir et Laâyoune.

\section{Méthodes}

Nous avons analysé la communication RSO à partir des sites internet et des réseaux sociaux officiels des cinq centres hospitaliers existants au Maroc (les liens sont disponibles en annexe).

Ainsi, nous avons relevé à partir de deux grilles d'analyse de site web médicaux (équipe régionale en documentation de la régie régionale de la santé et des services sociaux de montréal-centre ,1997) \& (bibliothèque de la faculté de médecine de l'université de liège, 2012) et d'une grille de communication RSO (Parker et al.,2010) les indicateurs compatibles aux domaines clés de notre étude sur la communication RSO dans le milieu hospitalier.

Il est à noter que malgré le fait que certaines grilles sont conçues pour supporter d'autres domaines, nous n’avons sélectionné que ce qui est adapté à notre étude, d'autres caractéristiques relatives au contexte marocain ont été rajoutées pour subvenir aux besoins de l'étude. La même démarche a été adoptée pour les réseaux sociaux, en tenant compte de certaines indications soulevées dans l'article "les réseaux sociaux: le nouveau défi de la communication institutionnelle hospitalière» (Aguerrebere,2012).

Nous tenons à préciser que les sites web et réseaux sociaux analysés sont relatifs au centre hospitalier dans son ensemble, c'est à dire qu'il n'existe pas de site web et/ou réseau social propre à chaque formation hospitalière ( entité hospitalière distincte : voir tableau $n^{\circ} 1$ ), seules des rubriques spécifiques relatives à ses formations sont disponibles sur le site global du centre hospitalier et renseignent sur ses formations par des presentations succintes et des descriptions sur leurs réalisations.

Pour ce qui est des réseaux sociaux, nous n'avons relevé que les liens officiels des réseaux en question et sont ceux qu'on retrouve généralement sur le site web d'un centre hospitalier; tout autre lien disponible sur la toile et relatif à une formation hospitalière quelconque ne peut être pris en considération.

La description de la grille d'analyse de sites web et réseaux sociaux comme le montre le tableau suivant-contient deux volets; le premier relatif à la localisation du terme responsabilité sociale/sociétale et ses formes de présentation sur le site/réseaux sociaux, et le deuxième contient la description du contenu communiqué sur les sites web/réseaux sociaux par les centres hospitaliers sur la RSO. 


\begin{tabular}{|c|c|c|}
\hline & \multicolumn{2}{|c|}{ Communication RSO } \\
\hline \multirow{4}{*}{$\begin{array}{l}\text { Structuration des } \\
\text { informations sur la } \\
\text { RSO sur le site } \\
\text { web }\end{array}$} & $\begin{array}{l}\text { Présence du terme } \\
\text { RSO/RSE sur le site }\end{array}$ & \\
\hline & $\begin{array}{l}\text { Localisation des } \\
\text { informations sur la } \\
\text { RSO }\end{array}$ & $\begin{array}{l}\text { (page d'accueil; rubrique;sous-rubrique;non } \\
\text { visible;non disponible;...) }\end{array}$ \\
\hline & $\begin{array}{l}\text { Formes de présentation } \\
\text { des informations sur la } \\
\text { RSO }\end{array}$ & $\begin{array}{c}\text { (texte/ multimédia: } \\
\text { vidéo;photos;animation;.../liens :pdf, word, } \\
\text { page web, liens externes/liste/base de } \\
\text { données/répertoire...) }\end{array}$ \\
\hline & $\begin{array}{c}\text { Formes/Outils de } \\
\text { communication sur la } \\
\text { RSO disponibles sur le } \\
\text { site }\end{array}$ & $\begin{array}{c}\text {-Bulletins d'information ; } \\
\text {-Revues de presse électronique ; } \\
\text {-Communiqués de presse ; } \\
\text {-Rapports/bilans d'activité ; } \\
\text {-Brochures/plaquettes institutionnelles/ } \\
\text { guides ; } \\
\text {-Audio ; } \\
\text {-Photothèque ; } \\
\text {-Vidéothèque ; }\end{array}$ \\
\hline \multirow{3}{*}{$\begin{array}{l}\text { Contenu RSO sur } \\
\text { le site } \\
\text { web/réseaux } \\
\text { sociaux }\end{array}$} & Responsabilité sociale & \multirow{3}{*}{$\begin{array}{l}\text { Actions RSO au profit du personnel, } \\
\text { médecins, et patients }\end{array}$} \\
\hline & $\begin{array}{c}\text { Responsabilité } \\
\text { environnementale }\end{array}$ & \\
\hline & $\begin{array}{l}\text { Responsabilité } \\
\text { économique }\end{array}$ & \\
\hline
\end{tabular}

Tableau 2 : Grille d'analyse de la communication RSO au sein des centres hospitaliers marocains

\section{Résultats}

Les résultats auxquels ont abouti nos analyses sont répartis comme suit :

\section{Analyse de la communication RSO sur les sites web officiels des cinq centres hospitaliers marocains}

$\mathrm{Au}$ début, il est à noter que notre recherche de l'expression "responsabilité sociétale/sociale » n’a pas abouti sur les sites web des centres hospitaliers étudiés, en effet, ce terme n'existe pas mais les actions/événements/informations y afférents existent, et apparaissent en majorité sur la page d'accueil des sites, il n’y a pas de rubriques spécifiques à ce propos, les informations communiquées diffèrent d'un centre hospitalier à un autre, cela confirme le fait que les hôpitaux font de la RSO sans se rendre compte.

Nous avons ainsi relevé que les informations liées à la RSO sont communiquées sur les sites web en question, sous divers formats : 
$\checkmark$ Affiches des manifestations et événements organisés par le $\mathrm{CH}$ ou l'un de ses établissements ou départements, brochures, plaquettes institutionnelles, guides,... ;

$\checkmark$ Enregistrements audio, photos et vidéos relatives aux événements sociaux organisés par le $\mathrm{CH}$ ou l'un de ses établissements ou départements qu'on retrouve soit répandus sur le site, soit dans les photothèques ou vidéothèques ;

$\checkmark$ Rapports d'activité, rapports annuels,rapports sur le développement durable...;

$\checkmark$ Bulletins/letttres d'information;

$\checkmark$ Volet relations avec la presse,on retrouve: articles de journaux numérisés, revues de presse en ligne, communiqués de presse, ...etc

En ce qui concerne le volet contenu, nous l'avons réparti selon les besoins de l'étude en trois grandes résponsabilités:

\section{Responsabilité sociale}

Dans une quête de durabilité et d'amélioration de la qualité et des conditions de soins et de services délivrés aux personnels et patients des centres hospitaliers, s'inscrit une responsabilité sociale hospitalière qui favorise la promotion de la santé sur plusieurs axes :

-Développement des compétences et des connaissances du personnel à travers des formations :

Nous avons noté une certaine richesse et variations des thèmes abordés et qui touchent plusieurs domaines liés à la RSO : (exemple du $\mathrm{CH}$ Ibn Sina : formations préparatoires aux examens d'aptitude professionnelle en rapport avec les thématiques RSO et en faveur du personnel et du corps enseignant: (les déterminaux sociaux de la santé, santé environnement et développement, la gestion des déchets médicaux et pharmaceutiques, la médiation et gestion de conflits,..etc));(exemple du $\mathrm{CH}$ Hassan II : formations sur l'utilisation des plates-formes de recherches bibliographiques et cliniques ); (des séances de formation au profit des infirmiers étrangers au $\mathrm{CH}$ Ibn Sina et organisation d'une cérémonie de fin de formation en leur honneur), cela vise à renforcer la cohésion sociale et les liens de solidarité et de fraternité avec les peuples étrangers.

-Politique ressources humaines ouverte et équitable:

A travers le lancement des avis de concours de recrutement, des avis d'examen professionnel, les annonces des résultats, le tout dans la transparence et un esprit de crédibilité.

-Renforcement de la cohésion sociale :

Plusieurs actions d'ordre social sont programmées au profit du personnel, enseignants et patients des centres hospitaliers :

$\checkmark$ Au profit du personnel : 
Célébration des journées internationales : journée de la femme, fête des retraités,..., colonie de vacances au profit des enfants des professionnels, etc...

$\checkmark$ Au profit des patients :

Actions telles que : course pour le don d'organe, soirée musicale à l'hôpital d'enfants au CH Ibn Rochd, amélioration de la qualité et sécurité des soins délivrés aux patients, à ce propos le centre hospitalier Ibn Rochd a reçu une attestation de participation au programme d'accréditation du service de psychiatrie, initié par le ministère de la santé marocain en 2014.

Mise en ligne d'informations utiles par exemple pour le cas du $\mathrm{CH}$ Hassan II : (Les sites d'urgence: adresses et téléphones 24h/24 ,7j/7, plan d'accès de l'hôpital, recommandations sur les visites, informations sur les consultations et hospitalisations: liste des médecins avec l'emploi du temps de chacun, annonces d'interdiction de fumer...

$\checkmark$ Au profit des médecins enseignants :

Approche participative: réunions avec le corps professoral et la direction des centres hospitaliers, discussions des plans d'actions, résolutions, propositions en vue d'amélioration des conditions de travail...

\section{Responsabilité environnementale}

La responsabilité environnementale est basée sur des axes environnementaux, ou en harmonie avec l'environnement.

Toute gestion des risques environnementaux s'avère être indispensable pour le bon fonctionnement de toute entité quelconque, en ce qui concerne les centres hospitaliers plusieurs actions sont entreprises tels que :

$\checkmark$ L'organisation de formations en matière de responsabilité environnementale (exemple du CH Ibn Sina : formations en santé environnement, gestion des déchets médicaux et pharmaceutiques,...);

$\checkmark$ La construction d'un site dédié au traitement des déchets d'activité de soins à risque infectieux au sein du CH Hassan II de Fès;

$\checkmark$ Réception d'attestations et de prix, suite à des actions dans le cadre environnemental : cas du CHU Mohammed VI de Marrakech qui a reçu au marge de la COP22, -événement international sur les changements climatiques organisé au du 7 au 18 novembre 2016 à Marrakech au Maroc , des certificats et prix de mérite; résultat de son engagement vert : sur la réduction de l'emprunte carbone, sur l'organisation d'événements à la marge de la cop 22 au profit des établissements de santé , prix gold « climate leadership », prix silver « climate resilency»... 
$\checkmark$ Dans le cadre de la gestion des déchets médicaux et pharmaceutiques, des opérations de (tri, collecte, transport, traitement et élimination) sont menées avec les sociétés de nettoyages, les communes urbaines, ou sous-traités à des sociétés spécialisées dans la gestion des déchets. Le degré de nuisibilité de ces déchets varie d'une catégorie à une autre, le $\mathrm{CH}$ Mohammed VI à Oujda les a répartis comme suit : déchets à risque d'infection ;organes et tissus non identifiables ; produits et dérivés sanguins à usage thérapeutique, incomplètement utilisés, avariés ou périmés; matériel piquant ou tranchant ; médicaments ;produits chimiques et biologiques, non utilisés, avariés, périmés; déchets cytostatiques et cytotoxiques;organes et tissus aisément identifiables par un non spécialiste ; déchets assimilés aux déchets ménagers,etc,... (CLIN du CH Mohammed VI d'Oujda,2015)

Ce type d'actions en faveur de l'environnement augmente l'engagement des établissements de santé et accentue leur présence sur le plan de responsabilité environnementale.

\section{Responsabilité économique}

La responsabilité économique est la moins communiquée de toutes les responsabilités citées auparavant, elle s’intéresse à la promotion d'une politique d'achat efficiente et responsable, l'intégration des principes d'éthique (exemple : existence d'un comité d'éthique au $\mathrm{CH}-\mathrm{Fès),} \mathrm{ainsi} \mathrm{que}$ les principes de déontologie et d'écologie, le tout dans un souci de rigueur et d’équilibre budgétaire.

Il s'agit d'une responsabilité qui relève en grande partie du fonctionnement interne de l'organisme, chose qui explique la faible communication des actions entreprises dans ce sens.

Reste à préciser que toute responsabilité sociale se doit d'être vertueuse économiquement afin de permettre l'accroissement des performances d'exploitation de toute organisation (Portail économique du val d'essonne, 2014).

On peut prendre comme exemple le fait de renseigner les gens en donnant le plus d'informations possible sur le site web afin de leur faire éviter des déplacements inutiles et parfois même coûteux en vue d'avoir une information quelconque qu'on pourrait facilement se procurer par internet ou par téléphone, cela permettra de faire des économies de transport, d'argent et de pollution. Peut être ajoutée à cela l'informatisation des dossiers des clients et l'opération de recyclage garants d'énergie et d’économie.

Sans prétendre à l'exhaustivité, les centres hospitaliers regorgent d'actions d'ordre social, environnemental ou économique, actions qui serviront de feuille de route pour tout établissement hospitalier responsable. 


\section{Analyse de la communication RSO sur les réseaux sociaux officiels des cinq centres hospitaliers marocains}

De nos jours chaque organisme présent sur la toile et disposant d'un site internet s’évertue d’être présent également sur les réseaux sociaux, avec des millions d'adeptes dans le monde,les réseaux sociaux sont devenus des outils incontournables de communication, de diffusion et de partage d'information.

Suite à cette révolution exceptionnelle, et vu que 82,1\% d'internautes marocains utilisent quotidiennement les réseaux sociaux (Agence nationale de réglementation des télécommunications, 2015), nos cinq centres hospitaliers marocains ont également pris part à cette démarche mais avec un taux très faible.

\begin{tabular}{|c|c|c|c|c|c|c|}
\hline & & $\begin{array}{l}\text { CH } \\
\text { Rabat } \\
\text { (Ibn } \\
\text { Sina) }\end{array}$ & $\begin{array}{c}\mathrm{CH} \\
\text { Casablanca } \\
\text { (Ibn Rochd) }\end{array}$ & $\begin{array}{c}\mathrm{CH} \\
\text { Marrakech } \\
\text { (Mohammed } \\
\text { VI) }\end{array}$ & $\begin{array}{c}\text { CH Fès } \\
\text { (Hassan } \\
\text { II) }\end{array}$ & $\begin{array}{c}\text { CH Oujda } \\
\text { (Mohammed } \\
\text { VI) }\end{array}$ \\
\hline \multirow{3}{*}{$\begin{array}{l}\text { Réseaux } \\
\text { sociaux } \\
\text { officiels } \\
\text { recensés }\end{array}$} & Facebook & \multirow{3}{*}{ Aucun } & \multirow{3}{*}{ Aucun } & $*$ & \multirow{3}{*}{ Aucun } & * \\
\hline & Twitter & & & $*$ & & \\
\hline & Google+ & & & * & & \\
\hline
\end{tabular}

Tableau 3 : Liste des réseaux sociaux relatifs à chaque centre hospitalier marocain

En effet, et comme le montre le tableau ci-dessus: sur les cinq centres hospitaliers du royaume, seulement deux sont présents de façon officielle sur les réseaux sociaux, ainsi on trouve le $\mathrm{CH}$ Mohammed VI de Marrakech présent sur twitter, google+ et de manière très active sur facebook, et le $\mathrm{CH}$ Mohammed VI de Oujda présent uniquement sur la célèbre plateforme facebook.

Les autres $\mathrm{CH}$ sont présents mais de manière non officielle c'est-àdire que leurs comptes ou pages sont gérés par des personnes qui ont montré de l'intérêt pour l'organisme, mais qui ne sont ni affiliées ni associées officiellement aux $\mathrm{CH}$ en question.

Les deux profils facebook des centres hospitaliers étudiés sont riches et performants, les champs permettant de fournir des informations utiles sur l'organisme sont bien renseignés: adresse, plan d'accès, numéros de téléphone, photos, etc..., le fil d'actualité est toujours d'actualité, la fréquence de publication est régulière et varie selon le timing et la nature des éléments communiqués.

Pour ce qui de l'interaction avec les visiteurs virtuels, on peut dire qu'elle existe mais de façon modeste, chose qui peut être expliquée par le nombre des followers (nombre des personnes qui suivent la page: les « j'aime » selon le langage de la plate-forme facebook) $\mathrm{CH}$ Mohammed VI de Marrakech (4759 personnes qui suivent) et le $\mathrm{CH}$ Mohammed VI 
de Oujda (11 447 personnes qui suivent) (dernière date de consultation:28/02/2017), des nombres considérés très faible par rapport à la population desservie .

En ce qui concerne la nature des informations communiquées, le $\mathrm{CH}$ Mohammed VI de Oujda et le CH Mohammed VI de Marrakech communiquent depuis la création de leur pages officielles, des informations variées, ainsi on retrouve:

-Des informations adressées à leurs personnels à savoir : les vœux (fêtes religieuses, fêtes nationales, journées mondiales (exemples : journée mondiale de l'infirmier, journée mondiale de la femme,...) et condoléances; volet ressources humaines: annonces et résultats des concours internes et externes, dates des concours, annonces des journées d'accueil pour les nouvelles recrues, annonces syndicales,etc,...

-On publie également les affiches et photos des manifestations organisées par les $\mathrm{CH}$ : tables rondes, conférences, campagnes de sensibilisation et de dépistage des maladies, don de sang, don d'organe,

-On retrouve aussi des conseils sur la santé (exemple : conseils sur la bonne nutrition, sensibilisation contre le tabagisme, conseils pour prendre soins de ses reins...).

-Des soirées sont organisées au profit des enfants malades, des visites des stars nationaux et internationaux,...

On note aussi la présence d'annonces pour des initiatives remarquables telles que la vulgarisation des maladies dans langue maternelle (dialecte marocain) : exemple d'une rencontre tenue sur le jeûne, dialyse, diabète et les maladies cardiaux au sein du CH Mohammed VI de Oujda.

Les deux reseaux sociaux restants du $\mathrm{CH}$ Mohammed VI de Marrakech à savoir twitter et google+ sont plutôt inactifs.

Dans l'ensemble les informations communiquées sur les réseaux sociaux étudiés sont d'ordre social, ainsi cela affirme la forte présence du social au détriment de l'environnemental et de l’économique .

\section{Conclusion}

Les résultats obtenus ont montré que les réseaux sociaux sont non seulement des outils complémentaires mais aussi des outils de rediffusion, c'est-à-dire qu'on retrouve les nouvelles communiquées à la fois sur le site web du centre hospitalier en question et sur ses réseaux sociaux,s’ils existent.

Ces nouveaux médias de communication institutionnelle ont leurs propres règles communicationnelles,ils visent l'échange d'expériences de santé entre les patients, la participation des citoyens dans la vie de l'hôpital même virtuellement et priviligie la socialisation de l'institution hospitalière à travers l'approche pédagogique plutôt que l'approche commerciale (lorsqu'il s’agit d'une institution privée) (Aguerrebere, 2012). 
Outre les éléments avancées ci-dessus, on peut dire aussi que pour notre cas l'interaction avec les visiteurs virtuels marocains demeure modeste et minime malgré le fait que les réseaux sociaux et les sites web sont considéré parmi les outils de communication les plus sollicités de l'ère numérique actuelle.

A ce propos, il est à noter que malgré cette revendication continue, des analphabètes demeure parmi les principales clientèles des établissements de santé publics, - le taux d'analphabétisme au Maroc s’avère être élevé, touchant environ $32 \%$ de la population totale- (Agence nationale de lutte contre l'analphabétisme, 2016) les personnes âgées et les gens avec un faible taux de revenu ou ne disposent pas de ressources suffisantes pour faire face aux dépenses inhérentes aux soins (programme RAMED) (Régime d'assistance médicale, 2011) sont loin d'être une population à desservir par les outils de communication numérique «les nouveaux programmes de communication de santé qui utilisent les réseaux sociaux en ligne doivent considérer, avant tout, l’âge de la population ciblée » (Chou et al. 2009) .

Pour y remédier une communication directe sur terrain pourra orienter beaucoup mieux cette catégorie vulnérable.

\section{Recommandations}

Nous recommandons fortement que chaque formation hospitalière (Hôpital/Centre) dispose de son propre site web, permettant de réunir toutes les informations et renseignements qui lui sont relatifs y compris les informations sur la responsabilité sociétale. Cela permettra aux visiteurs un meilleur accès et une navigation plus facile et rapide pour pouvoir accéder au contenu souhaité, au lieu de se perdre dans des informations dissipées sur un site web général, comme ce fût le cas pour les sites web étudiés.

Il faudra également à l'instar des hôpitaux internationaux, instaurer une politique RSO propre à chaque hôpital, ou propre au centre hospitalier dans sa globalité, cette politique et en vue d'une bonne communication pourra ainsi figurer sur le site web officiel dans une rubrique spécifique dédiée à la RSO .

Mis à part les centres hospitaliers Ibn Sina de Rabat,Mohammed VI de Marrakech et Hassan II de Fès, nous recommandons une refonte des sites web des centres hospitaliers restants et d'assurer une meilleure interaction via les réseaux sociaux pour l'ensemble des centres hospitaliers étudiés.

La pérennisation des actions faites en matière de RSO ne pourra avoir lieu qu'à travers une communication numérique à jour, organisée et réciproque. 


\section{References:}

1. Agence nationale de réglementation des télécommunications (2014). 11ème enquête nationale annuelle sur les Tic auprès des ménages et des individus

2. Agence nationale de réglementation des télécommunications (2015). Enquête annuelle sur l'accès et l'utilisation des technologies de l'information et de la communication (TIC)

3. Aguerrebere, P. M. Les réseaux sociaux: le nouveau défi de la communication institutionnelle hospitalière. http://w3. u-grenoble3. fr/les_enjeux/index. php, 107

4. B.O No 5388 DU 18 HIJA 1426 (19/1/2006).Arrêté conjoint du Premier ministre, du ministre de l'éducation nationale, de l'enseignement supérieur, de La formation des cadres et de la recherche scientifique et du ministre de la santé $n^{\circ} 2340-05$ du 1er hija 1426 (2 janvier 2006) fixant la composition des centres hospitaliers et universitaires.Repéré à : http://www.sante.gov.ma/Reglementation/etablissementssoustutelled uministeredelasante/2340-05.pdf

5. Bibliothèque de la faculté de médecine de l’université de liège.(2012) Grille d'analyse des sites médicaux sur l'Internet. Repéré à : http://www.ebm.lib.ulg.ac.be/prostate/grille.htm

6. Chou, W. Y. S., Hunt, Y. M., Beckjord, E. B., Moser, R. P., \& Hesse, B. W. (2009). Social media use in the United States: implications for health communication. Journal of medical Internet research, 11(4), e48.

7. CLIN-CHU mohammed VI oujda (2015).Gestion des déchets médicaux et pharmaceutiques. Repéré à http://www.chuoujda.ma/clin/1.pdf

8. Dahir $\mathrm{n}^{\circ} 1.82 .5$ du 30 rebia i 1403 (15 janvier 1983) portant promulgation de la loi $\mathrm{n}^{\circ} 37.80$ relative aux centres hospitaliers Référence : B.O N 3668 du 16 Février 1983

9. Décret $\mathrm{n}^{\circ}$ 2-04-776 du 14 kaada 1425 relatif aux centres hospitaliers et universitaires (B.O. $\mathrm{n}^{\circ} 5280$ du 6 janvier 2005). Référence : Bulletin officiel $n^{\circ} 5280$ du 6 janvier 2005

10. Équipe régionale en documentation, Régie régionale de la santé et des services sociaux Montréal-Centre.(1997). Grille d'analyse de sites Web. Repéré à : http://publications.santemontreal.qc.ca/uploads/tx_asssmpublications/ pdf/publications/1997_grille_web.pdf

11. Martel,F.(2014). La responsabilité sociétale pour les nuls : qu'est-ce que la RSE ? Comment mettre en œuvre dans son établissement une politique de RSE ?. Repéré à http://www.fhf.fr/Developpement- 
durable/Documents-de-reference/RSE-La-responsabilite-societalepour-les-nuls

12. Ministère de l’Intérieur (2012). Régime d’assistance médicale. Repéré https://www.ramed.ma/(S(40wifq0fctiqrhkxnazgbaou))/fr/SInformer/ ConditionsAdhesion.aspx

13. Organisation Mondiale de la Santé.(1997). Déclaration de Jakarta sur la promotion de la santé au XXIe siècle. Repéré à http://www.who.int/healthpromotion/conferences/previous/jakarta/en/ hpr_jakarta_declaration_fr.pdf

14. Parker, C. M., Zutshi, A., \& Fraunholz, B. (2010, January). Online corporate

15. social responsibility communication by Australian SMEs: a framework for

16. website analysis. In Bled 2010: Proceedings of the 23rd Bled eConference:

17. eTrust: implications for the individual, enterprises and society (pp. 509-

18. 523). University of Maribor

19. Portail économique du val d'essonne.(2014). La responsabilité sociale et environnementale : un enjeu majeur pour les entreprises.Repéré à : http://www.valdessonne-economie.com/dossiers/326-laresponsabilite sociale-et-environnementale-un-enjeu-majeur-pourles-entreprises

\section{Annexes :}

Liens des sites web et réseaux sociaux officiels analysés-dernière consultation le : 28/02/2017 :

\begin{tabular}{|c|c|c|}
\hline $\begin{array}{c}\text { Centres } \\
\text { Hospitaliers }\end{array}$ & Sites web & Réseaux sociaux \\
\hline $\begin{array}{l}\text { CH Rabat } \\
\text { (Ibn Sina) }\end{array}$ & www.chisrabat.ma & Inexistent \\
\hline $\begin{array}{c}\mathrm{CH} \\
\text { Casablanca } \\
\text { (Ibn Rochd) }\end{array}$ & www.chuibnrochd.ma & Inexistent \\
\hline $\begin{array}{c}\mathrm{CH} \\
\text { Marrakech } \\
\text { (Mohammed } \\
\text { VI) }\end{array}$ & $\begin{array}{c}\text { www.chumarrakech. } \\
\text { ma }\end{array}$ & $\begin{array}{c}\text { Facebook: } \\
\text { https://www.facebook.com/chumohammedvi/ } \\
\text { Tweeter : https://twitter.com/chumarrakech }\end{array}$ \\
\hline $\begin{array}{l}\text { CH Fès } \\
\text { (Hassan II) }\end{array}$ & www.chu-fes.ma & Inexistent \\
\hline $\begin{array}{l}\text { CH Oujda } \\
\text { (Mohammed } \\
\text { VI) }\end{array}$ & www.chuoujda.ma & $\begin{array}{c}\text { Facebook: } \\
\text { https://www.facebook.com/CentreHospitalierUniversitaire } \\
\text { MohammedViOujda/ }\end{array}$ \\
\hline
\end{tabular}

